\section{Intraoperative visualization of the aortic valve from the left ventricle}

\author{
Akira Furuse, MD, Tadasu Kohno, MD, and \\ Toshiya Ohtsuka, MD, Tokyo, Japan
}

To evaluate the degree of competence achieved after aortic valve repair, Ghandour, Halees, and Duran ${ }^{1}$ introduced a fiberoptic cystoscope into the ascending aorta filled with cardioplegic solution.

For intraoperative assessment of aortic regurgitation, we believe the reverse approach from within the left ventricle is much more informative than the aortic approach. The purpose of this communication is to describe our method of video-assisted visualization of the ventricular surface of the repaired aortic valve. The scope we are using is a fiberoptic thoracoscope, the tip of which can be bent to 60 degrees upward and 130 degrees downward (LTF fiberacope; Olympus Optical Co. Ltd., Tokyo, Japan).

After the completion of the aortic repair, the thoracoscope is introduced into the left ventricle in the following way. The right atriotomy is made after both cavae are snared. The thoracoscope is introduced into the left atrium through a small incision in the fossa ovalis. While observing the video screen, we manipulate the flexible scope and advance it into the left ventricle across the mitral valve, which is clearly visible through the scope. Effective left atrial venting through a tube inserted from the right upper pulmonary vein is important at this stage of the procedure. By further manipulation, the scope can be placed directly beneath the aortic valve. The exact location of the tip of the scope is confirmed by direct visualization through the aortotomy.

The aorta is closed with two continuous mattress sutures started at both extremities of the aortotomy. Two arms of sutures are approximated by placing them through a keeper. Cold crystalloid cardioplegic solution is administered into the ascending aorta through an aortic vent tube. This fluid fills the aortic root while the aortotomy keeper is loosened and both coronary arteries are pressed by fingers to avoid air embolism. The repaired aortic valve and any regurgitant jet can be seen on the video screen and recorded on the videotape (Fig. 1).

Once the viewing is completed and the repair is seen to be satisfactory, the scope is removed. After two arms of the aortic mattress sutures are tied, additional running sutures from both extremities of the aortotomy are completed. The aorta is unclamped with the aorta vented for suction of any residual air. If the repair is found to be unsatisfactory, further surgical maneuvers are undertaken, with the scope left inside the left ventricle.

From the Department of Cardiothoracic Surgery, University of Tokyo Hospital, Tokyo, Japan.

J ThoraC CARDIOVASC SuRg 1995;109:395

Copyright $(1995$ by Mosby-Year Book, Inc.

0022-5223/95\$3.00+ $0 \quad \mathbf{1 2 / 8 / 5 8 6 1 5}$

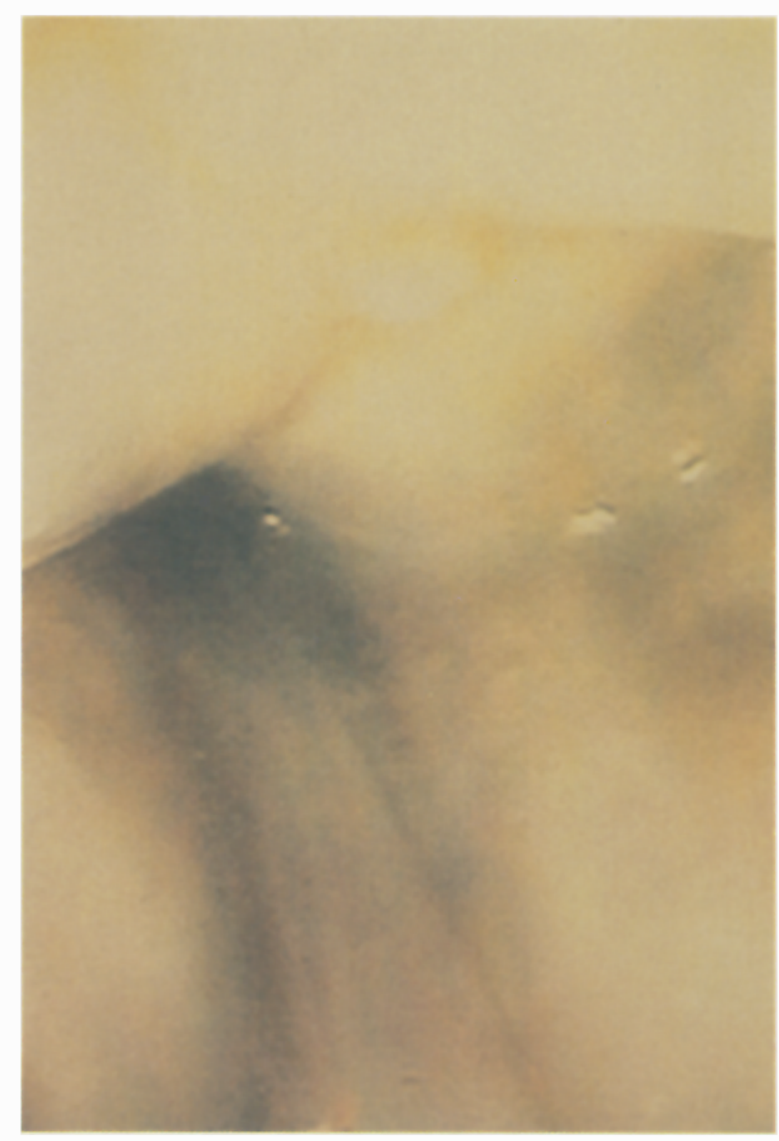

Fig. 1. Left ventricular view of the aortic valve through the scope. The completent but slightly uneven surface of aortic cusps can be seen.

After we became accustomed to video-assisted thoracoscopic surgery for intrathoracic diseases, ${ }^{2}$ the use of the scope was extended to visualization of cardiovascular structures during cardiopulmonary bypass. This method has been applied to more than 10 patients without any obvious problems. The technique is particularly effective to evaluate aortic valve competency before the release of the aortic crossclamp.

\section{REFERENCES}

1. Ghandour M, Halees ZA, Duran C. A simple method for intraoperative visualization of the repaired aortic valve [Letter]. J Thorac Cardiovasc Surg 1994;107: 632-4.

2. Kohno T, Murakami T, Wakabayashi A. Anatomic lobectomy of the lung by means of thoracoscopy. J Thorac Cardiovasc Surg 1993;105:729-31. 